\title{
Ocular Surface Disease: An Integral Part of Glaucoma Management
}

\author{
Aysha Salam ${ }^{1}$ \\ ${ }^{1}$ Consultant Ophthalmologist, Royal Liverpool Hospital, United Kingdom
}

Glaucoma has been a silent and symptomless diseasecausing relentless loss of vision requiring lifelong treatment. It is often hard to gain patient acceptance for a condition, the treatment for which does not manifest in the form of any direct visual gains. This is in turn is compounded by the adverse effects of medication. These are most commonly poor tolerance and preservative toxicity in addition to the active ingredient which in itself can compromise a vulnerable ocular surface. ${ }^{1,2}$ Glaucoma patients are presumably at a much higher risk of developing ocular surface disease and one of the commonest reasons for that is being treated with preservative-containing medications over a prolonged period. ${ }^{1,2}$ This editorial will highlight some of the common risk factors and possible remedies in glaucoma patients manifesting with ocular surface disease.

Preservative toxicity is a well-known concept but is still under diagnosed and poorly managed in most patients. Benzalkonium Chloride (BAK) is the most commonly used preservative, in most ophthalmic preparations. The other preservatives used are Polyquad and peroxide derivatives. ${ }^{3}$ The main benefit of using preservatives is to prevent bacterial contamination along with the requirement by the regulatory authorities for a safe dispensing of the medication. ${ }^{4,5}$ However, the BAK is known to induce necrosis (at conc. of $0.05-0.1 \%$ ) and cellular apoptosis (at conc. of $0.01 \%$ ) by disturbing cellular membrane in bacterial cells. Human ocular surface

How to Cite this Article: Salam A. Ocular Surface Disease: An Integral Part of Glaucoma Management. Pak J Ophthalmol. 2020; 36 (2): 194-196.

Doi: $10.36351 /$ pjo.v36i3.1064

Correspondence to: Aysha Salam

Consultant Ophthalmologist, Royal Liverpool Hospital

Email: Aysha.salam320@gmail.com cells absorb BAK, which has damaging effects similar to those in bacterial cells. They are cumulative and become more severe with more concentrated and frequent exposures. The inflammatory changes induce permanent damage to the conjunctival goblet cells, which can seriously compromise the success of any future filtration procedures. ${ }^{3,4,5}$

In order to fully analyse the histological and morphological changes in patients treated with BAK containing glaucoma drops, a prospective, doublemasked, placebo-controlled study was performed by Barabino et al who assessed corneal thickness, goblet cell density and conjunctival immunohistochemistry using CD45 detection in rats at day 7 of treatment. ${ }^{6}$ The study revealed higher incidence of punctate fluorescein staining in BAK treated eyes as compared to the control group although the changes in corneal thickness were not statistically significant. The BAKtreated eyes showed a significantly lower number of Goblet cells than those in all control groups $\left({ }^{*} P<\right.$ 0.05 ), whereas it induced a significant increase in the CD45 expression in the bulbar conjunctiva showing an increase in the inflammatory activity. ${ }^{6}$

The significance of this is further endorsed in a study by Agnifil et $\mathrm{al}^{7}$, who analysed the pre-operative conjunctival goblet cell density (GC), MUC5AC which is the main mucin product of GC, and HLA-DR in glaucomatous patients undergoing trabeculectomy, using laser scanning confocal microscopy (LSCM) and impression cytology (IC). They divided their patients into three groups. The group with complete success revealed good population of goblet cells with scattered microcysts showing a functioning bleb. The impression cytology also confirmed MUC5AC being significantly over expressed compared to failed surgeries along with a better IOP control. The groups with qualified success and failed trabeculectomies had much lower Goblet cell densities and MUC5AC concentrations although the inflammatory component 
HAL-DR was not statistically different in the three groups. $^{7}$

The study establishes the most important myths behind a successful filtration procedure which would appear to be a favourable density of the goblet cells which could in future be used as a biomarker to predict the chances of success of surgery and post-operative bleb management. A decrease in the concentration of Goblet cells with increase in mast cells, squamous metaplasia, dendritic cell proliferation and fibroblasts would be the most likely progenitors for scarring and surgical failure. ${ }^{6,7}$.

Glaucoma is common in the elderly and due to chronicity, is more likely to require more frequent use of multiple topical medications which precipitate the risk of Ocular surface disease. The underlying pathophysiology includes functional changes with reduced function of the lacrimal glands causing aqueous tear deficiency with under function of the meibomian glands as well as reduced number of conjunctival goblet cells. There is often secondary inflammation of the ocular surface as a result of meibomian gland dysfunction which can in turn blunt the effect of glaucoma medications causing poor control and intolerance to glaucoma drops. The ageing of the ocular Adenexa with lid laxity, pump failure and conjunctivochalasis are other risk factors common in this age group, which further predispose to ocular surface disease ${ }^{8}$.

Early onset glaucoma most commonly requires surgical intervention as it is more aggressive and harder to treat. However, given that the disease process persists through an extended period of time, younger patents are likely to be exposed to topical medications for longer length of time and are more likely to suffer from cumulative toxicity of the preservatives ${ }^{9}$.

It is well known that dry eyes and OSD is much more common in older women particularly in the postmenopausal age groups. The role of Hormone replacement therapy (HRT) in improving the OSD is not well established, as the bulk of the problem is loss of circulating androgens, which are not given as a part of HRT. The largest cross-sectional study to date showed increase in the risk and severity of OSD in women. ${ }^{10}$

There is a wide range of medical and ocular conditions associated with increased risk of Ocular surface disease ${ }^{11}$. A detailed history is therefore crucial.

Medical illnesses include Collagen Vascular disorders, Chronic Graft Versus Host Disease (GVHD) after stem cell transplantation only seen in Allogenic transplantation, Androgen insufficiency, Iatrogenic factors including; Chemotherapy and Immunosuppressive therapy, Anti-Histamines and Antidepressants, Beta-blockers, Anti-Androgens, Isotretinoin, Radiation therapy and any Ocular surgery can spark dry eye disease.

It is recommended to discontinue BAK containing medicines and substitute with preservative free Medications or in severe cases, stop medications altogether and substitute with oral Acetazolamide.

Take home message is liberal use of preservative free medications, early SLT or Primary treatment, consider early surgery where there is disc damage and progression, pre-perimetric glaucoma progression, poor compliance, poor response to treatment and poor ocular surface.

It is evident that protecting the integrity of the ocular surface is as crucial as is treating glaucoma because without a healthy ocular surface, glaucoma management is bound to fail. Glaucoma care pathway is essentially incomplete without the ocular surface optimization. Early detection and treatment will predict a smoother journey with a successful long term outcome.

\section{Conflict of Interest}

Author declared no conflict of interest

\section{REFERENCES}

1. Baudouin C, Labbé A, Liang H, Pauly A, BrignoleBaudouin F. Preservatives in eye drops: the good, the bad and the ugly. Prog Retin Eye Res. 2010; 29 (4): 312-334.

2. Leung E, Mendeiros F, Weinreb R. Prevalence of Ocular Surface Disease in Glaucoma patients. Journal of Glaucoma, 2008; 17 (5): 350-355.

3. Freeman, $\mathbf{P}$ and Kahook M. Preservatives in topical Medications. Historical and clinical perspectives. Expert Rev Ophthalmol. 2009; 4 (1): 59-64.

4. Maiti S., Sadhukhan S., Bakshi P. Ocular Preservatives: Risks and Recent Trends in Its Application in Ocular Drug Delivery (ODD). In: Pathak Y., Sutariya V., Hirani A. (eds). Nano- 
Biomaterials For Ophthalmic Drug Delivery. Springer, Cham. 2016.

5. Jean MDS, Brignole F, Bringuier AF, Bauchet A, Feldmann G, Baudouin C. Effects of benzalkonium chloride on growth and survival of Chang conjunctival cells. Invest. Ophthalmol. Vis. Sci. 1999; 40 (3): 619630.

6. Barabino S, Antonelli S, Cimbolini N, Mauro V, Bouzin M. The Effect of Preservatives and Antiglaucoma Treatments on the Ocular Surface of Mice with Dry Eye. Invest. Ophthalmol. Vis. Sci. 2014; 55 (10): 6499-6504.

7. Agnifili L, Fasanella V, Mastropasqua R, Frezzotti $\mathbf{P}$, Curcio C, Brescia $\mathbf{L}$, et al. In Vivo Goblet Cell Density as a Potential Indicator of Glaucoma Filtration
Surgery Outcome. Invest. Ophthalmol. Vis. Sci. 2016; 57 (7): 2928-2935.

8. Ding J, Sullivan DA. Aging and dry eye disease. Exp Gerontol. 2012; 47 (7): 483-490.

9. Huang W. Pediatric glaucoma: A review of the basics, 2014. Available from:

http://https://www.reviewofophthalmology.com/article/ pediatric-glaucoma-a-review-of-the-basics

10. Sullivan DA, Rocha EM, Aragona P, Clayton JA, Ding J, Golebiowski B, et al. TFOS DEWS II Sex, Gender, and Hormones Report. Ocul Surf. 2017; 15 (3): 284-333.

11. Messmer EM. The pathophysiology, diagnosis, and treatment of dry eye disease. Dtsch Arztebl Int. 2015 Jan 30; 112 (5): 71-81. 